\title{
APPLICABILITY OF INTERNET VIDEO IN DISTANCE EDUCATION FOR ENGINEERING
}

\author{
J. Mark Pullen ${ }^{1}$
}

\begin{abstract}
This paper analyzes the pros and cons of Internet video in delivery of synchronous distance education for engineering topics and presents results of a recent experiment in adding video to a course that previously was delivered using Internet audiographics only. We have extensive experience teaching a variety of courses that support regional students by reducing commuting and also allowing time-shifting of classes using online recordings of the synchronous sessions. Our distance delivery had evolved away from video as a teaching medium in order to use limited capacity Internet modem links. With the recent availability of higher access rates using cable modem and DSL, video has become more practical as a teaching medium. This has led us to experiment with web-based video delivery. In this paper we provide a detailed analysis of the advantages and disadvantages of video when used in this way, and support the analysis with feedback provided by students in a controlled experiment where the same students attended classes with and without Internet video.
\end{abstract}

Index Terms: synchronous distance education, desktop audiographics, Internet video

\section{INTRODUCTION}

Beginning in 1994, the author has conducted an active research program in distance education at George Mason University (GMU). This work has maintained a very applied aspect in that it has been associated with presentation of one or more courses per semester to regional, off-campus students at their homes and offices. Results have been reported in [1], [3], [5], [6], [7] and [8]. Our earliest experiments used the Internet Mbone technologies [2] including audio, video, text and graphics. During the first year of this work we discovered that the educational effectiveness of real-time video delivery was significantly less than that of real-time graphics delivery if good quality audio was available in both cases. As the audio plus graphics (audiographics) with no video can be delivered over a readily available dialup modem link, we abandoned video delivery and focused on real-time audiographics from 1996 through 1999.

By Spring 2000, availability of compressed video technology and higher capacity connections had increased greatly over the situation in 1996. Internet video technology including the RealVideo system from RealNetworks provides usable video of moderate resolution and refresh rate at data rates under 100 kilobits per second. Many offices have Internet access at rates approaching or exceeding one megabit per second, while home access via Integrated Services Digital Network (ISDN), cable modem, or Digital Subscriber Line (DSL) offers service at rates above 100 kilobits per second. This motivated us to revisit the issue of video as a component of synchronous Internet course delivery. This paper provides a detailed description of the media issues involved and the lessons learned, including initial student feedback.

\section{MEDIA ROLES FOR INTERNET TEACHING}

Each of the media that can be used to teach over the Internet has specific characteristics making it valuable for some aspects of education. Here we consider those characteristics, in light of their educational roles.

- Text: This is the most basic form of computer communication. My colleague E. Norris has conducted several full courses in a Multi-User Virtual Environment or MUVE [7], groupware that offers a shared virtual space based entirely on shared text communication.

- Audio: While text "chat" can facilitate discussion among a dis tributed group, we have not found it to be an acceptable substitute for the spoken word. Many students are auditory learners. The audio component is, therefore, a cornerstone of distributed education.

- Static graphics: Other students are visual learners and all can benefit from having information presented in more than one form. The ability to display graphics with diagrams and a variety of text fonts is essential to the teaching environment.

- Graphic annotation: Graphics are made much more compelling if they can be annotated in real time in a way that is visible to all participants. This annotation might be used to add missing information, but often its function is simply to highlight the static graphic, drawing the student's attention to particular points.

- Motion video: It seems there is an inevitable link in the minds of educators between video and teleteaching. In point of fact, a much better application for video in teaching is playback of recorded material to illustrate the lecture [6]. The value of real-time video in synchronous distance education will be addressed further below.

\footnotetext{
${ }^{1}$ J. Mark Pullen, Department of Computer Science and C3I Center, George Mason University, Fairfax, VA 22030, mpullen@ gmu.edu
} 
- Immersion: Technology is available today to generate highly realistic virtual worlds that can be shared over the Internet [4]. High-resolution graphics, potentially in surround mode, can be coupled with computer graphics and networked virtual reality software to display the same views at multiple locations, where the actions of one participant are immediately evident to all others.

In principle, any of these media could be used to communicate among the students in addition to supporting communication between instructor and students. In practice, channels among students, and from student back to instructor, often are more limited than those from instructor to students. Also most Internet service does not yet offer adequate quality of service (QoS) to deliver real-time motion video and immersion. Figure 1 shows the space defined by the media described above, when both instructor presentation and student participation are considered. The issues in choosing appropriate media for lectures and seminars are (1) whether the available media are rich enough to support effective teaching and learning and (2) whether they are affordable in the Internet.

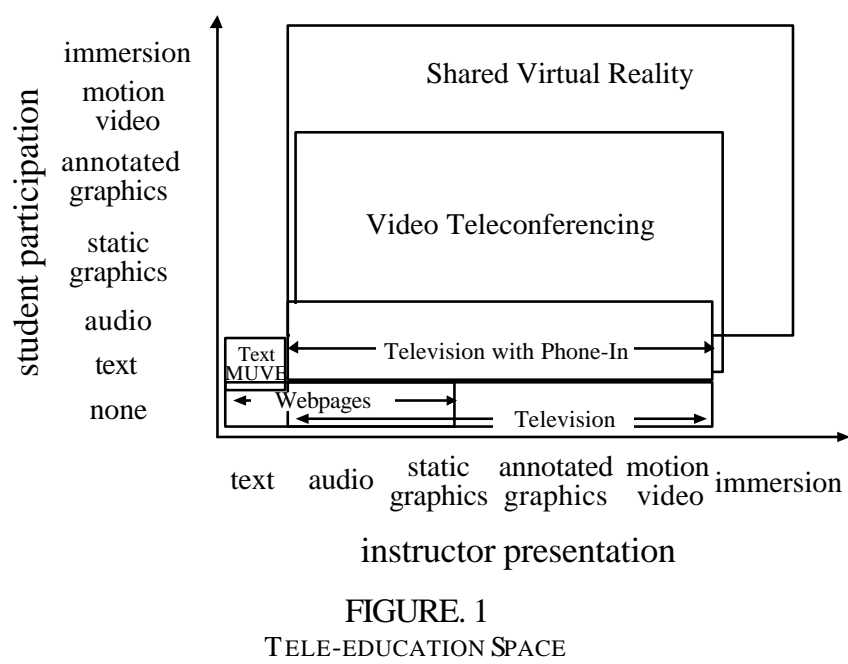

\section{MULTIMEDIA SYSTEMS FOR SYNCHRONOUS INTERNET TEACHING}

Two general approaches are in use today for synchronous Internet teaching. Both rely on the world-wide web (WWW) for initial user access, and both offer the possibility of repeating the educational session after its presentation, via recording and online playback. We describe these systems as webcasting and desktop audiographics.

- Webcasting uses a WWW browser with custom "plugin" software. The student can receive static graphics plus streaming audio and video. Major educational programs using webcasting are underway at institutions such as Stanford University (http://stanfordonline.stanford.edu) and the University of California at
Berkeley (http://bmrc.berkeley.edu). Streaming audio and video deal with the Internet QoS problem by buffering a significant amount of the data stream (typically at least 30 seconds) to avoid gaps in presentation due to stalls in network delivery.

- Desktop audiographics also involves a Web browser and plug-in, but communicates audio using Internet telephony (real time audio) and provides real-time annotations over pre-loaded static graphics. It deals with the QoS problem by operating at a low data rate that can, if necessary, be supported by dialup connection to a facility with adequate network capacity. As a result, it can support a truly synchronous lecture or seminar. Currently we are using the ClassWise desktop audiographic system [3], which has the additional feature that its classes can be recorded for asynchronous playback.

\section{EXPERIENCE IN DISTANCE EDUCATION WITHOUT VIDEO}

Reference [3] describes our experience in teaching with desktop audiographics that has led to the methodology currently followed by the author and several colleagues in the GMU Computer Science Department. Our basic approach has been to deliver our lectures online in audiographic media, available both synchronously and asynchronously. Our rationale for this is straightforward: The value of a teacher/mentor who continuously interprets the material being taught to the contemporary needs of the students is reinforced by centuries of educational tradition. Having no reason to doubt the effectiveness of this relationship, we have pursued synchronous distributed education since the beginning of our work.

We began our experiments in distributed education in 1994 using the Internet Multicast Backbone (Mbone) [2], which offers a capability to hold a shared audio/video/graphics session at multiple sites that are specially equipped to provide such services (typically, universities and laboratories). Figure 2 shows the configuration used to teach with the Mbone. As reported in [5], we discovered that the educational experience of remote students could be at least as good as that experienced in the local classroom, at some cost in additional preparation by the instructor. Our sample was small so we could not characterize the results statistically, but the students reported satisfaction with classes delivered. The ability to record the Mbone sessions for later playback also proved highly popular among working students who had missed some classes while travelling for their employers. 


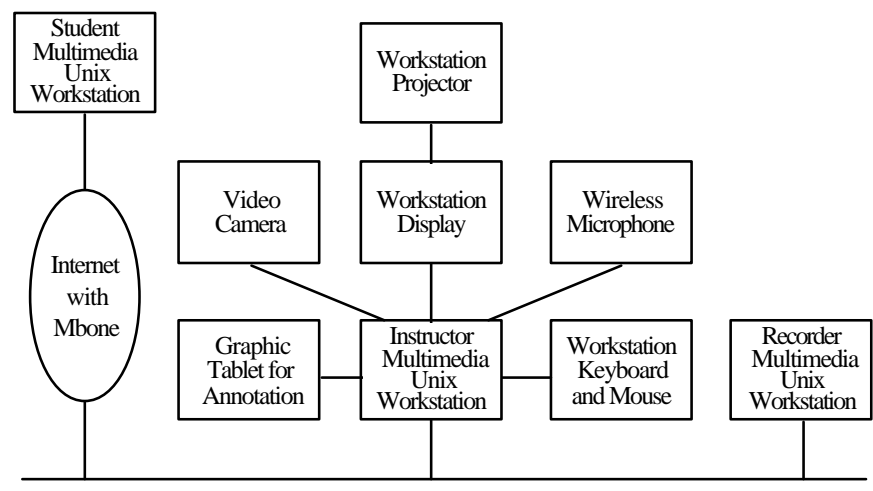

LAN with Mbone Support

FIGURE. 2

MBONE TEACHING CONFIGURATION (INCLUDING VIDEO)

We found Mbone distributed education to be very effective. However, at the time it was also a very expensive solution in terms of the terminal equipment requirements (Sun or Silicon Graphics workstations) and even more so in terms of the quality of Internet connection. Typically at least a 1.5 Megabit per second connection with multicast capability is required. We were seeking a means of distributed education to desktop facilities at home and office. Therefore, we needed a capability to deliver common platforms via network resources routinely available in these settings. Although the Mbone software now is available on Windows NT workstations, it still requires network facilities beyond a normal modem connection. Moreover, we had learned that the Mbone video we had assumed to be essential for distance education was in fact not essential. We did not have a camera operator or a "follow-me" camera for our Mbone teaching system. As a result, the instructor was offcamera most of the time, yet there were no student complaints. When asked, the students stated that they did not find the video essential to follow the class.

The element of the Mbone suite that we did find to be essential for effective presentations to distant students is real-time annotation of static graphics. The ability of the instructor to prepare slides in advance and then animate a presentation in real time by highlighting material, drawing on graphics to illustrate relationships and problem solutions, is central to our teaching methodology. We prepared slides in advance using commercial graphics software and displayed them on the Mbone whiteboard (WB) as a slide show. The whiteboard allows the slides to be annotated in real time with any of several colors. We found this capability to be far more useful in teaching technical subjects than camera-based video. The whiteboard produces a much clearer graphic image than video, while demanding only a small fraction of the network capacity required by video. This turned out to be the key to delivering a quality synchronous educational experience at modem data rates (we took the 28.8 kilobits per second modem as our standard).
Our second generation synchronous teaching technology was a product called ClassWise, which had roots in research conducted at GMU. The teaching configuration is shown in Figure 3. It transfers the following information in real time:

- the instructor's voice, transmitted using the standard GSM compression scheme developed for digital cellular telephony, at under 15 kilobits per second

- graphics whiteboard information for changing and annotating slides (the actual slides are transferred from the server asynchronously before class)

- text for "chat" style communication between the students and instructor

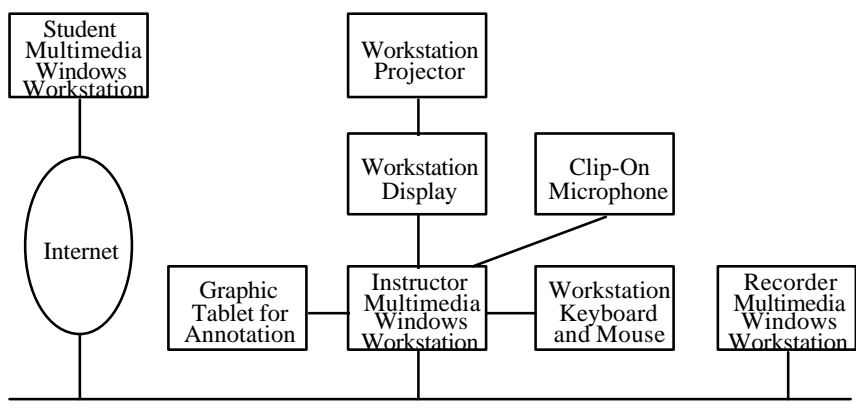

LAN connected to Internet

FIGURE. 3

DESKTOP AUDIOGRAPHIC TEACHING CONFIGURATION (NO VIDEO)

Students have reported repeatedly that classes delivered using only these media provide a good educational experience. Most students who have chosen to participate in class this way report that any loss from not being physically present in class is more than offset by the time and cost saving of not having to commute to class. However, teaching in this environment requires some techniques that are not required in "chalk talk" lectures. The factors of reduced communication (particularly the lack of "body language" feedback to the instructor) and lack of group rapport make the distributed teaching environment significantly different from the traditional classroom. Further, the substitution of a high-quality computer graphic display for live motion video requires some different presentation skills.

\section{Class Structure}

We have used this distributed education environment in four different modes:

- All students at remote desktops : We find that students typically respond positively to desktop delivery at first exposure. However, instructors require two or three classes to get used to teaching with no immediate feedback from students. After this time they settle in to a radio-announcer-like mode and adapt their delivery to the new media, as described below. 
- Asynchronous desktop students: The majority of our students are employed outside the university. They are quite busy during work hours and they miss many synchronous classes, which they make up in asynchronous mode. They value the mixed synchronous/asynchronous system highly for this flexibility.

- Classrooms at multiple campuses: In recent years, GMU offered distance education to students at a remote campus in parallel with delivery at our main campus, using large projected images obtained by commercial videoconferencing and whiteboard systems. We tried delivering only audiographics in place of this arrangement and found that we could not distinguish any difference in the students' response based on the lack of video.

- Mixture of multiple classrooms and desktop students: Once a desktop audiographic system was in use between campuses, it became possible for students at home or office to participate also. This option was elected by a small fraction of students with good success. The asynchronous mode was more popular than the synchronous, probably because the students had signed up for classroom participation and thus typically sought to use the desktop system only when they had to miss a class due to travel.

\section{Annotations}

A whiteboard can be annotated with lines, ellipses, rectangles, and other graphic symbols such as arrows. The geometric figures may be in any of several configurations of dashes, line widths, and colors. When placed on the instructor's whiteboard, these are transmitted to the student's whiteboard in real time. In addition to allowing dynamic creation of simple figures, the annotations are useful in drawing the student's attention to the part of the slide the instructor is discussing. Typical techniques are underscoring words, drawing boxes or ellipses around key words and figures, and placing arrows next to points as they are covered. The result is a dynamic graphic display that maintains interest for the visual learner. Instructors quickly gain facility with this capability and use it to enhance their presentations.

\section{Student Questions}

In our experience, student questions are few from any remote group whether in remote classrooms or at desktops. Part of the reason for this appears to be that the higher level of organization of the classes, coupled with the fact that the slides are available to students before class, leaves fewer points on which the students are in doubt. However, the greater psychological gap between instructor and remote student also seems to influence the students' willingness to ask questions. We have found that the practice of sprinkling a class session with questions, customary among many instructors of in-person classes, also works in the distributed education environment to engender better dialog and to give the instructor feedback as to how well the material is being assimilated by students. This sort of "mini quiz" engages the students in the class more fully, in addition to helping the instructor pace the presentation for best comprehension.

\section{Recording}

We have come to understand that the freedom to participate asynchronously is one of the features of distributed education that students value highly. The asynchronous mode also has made possible professional education delivery to smaller groups that would not justify formation of a new class. However, it has been our consistent experience that many students prefer the interactivity of synchronous presentation when conditions make it feasible.

\section{Student Evaluations and Grades}

GMU collects a course evaluation from students. Participation is anonymous and voluntary. In [3] we provide data from evaluations of distant and local students, where a total of 41 out of 68 students participated in the evaluation. The average of overall evaluations given by remote students was 4.12 while the average for the local class was 3.91 . Compared with a possible maximum of 5 and a departmentwide average of 4.09 , we interpret this as showing no significant difference between the two groups, or between the whole class and a typical class. Subsequent evaluations have produced similar results. With respect to grades, on a four-point scale $(A=4)$ the local students' average was 2.98 and the remote students' average was 3.07. Grade distribution was virtually identical in both cases. Based on grades there was no indication that distant students learned more or less than local students.

After publication of [3] we taught four more semesters using desktop audiographics, with similar results. Student enthusiasm was sufficiently high that in Fall 2000 we offered two sections for one course: a classroom section and a "NET" section. Our approach falls short of full distance education in that exams must be taken locally. While both sections have the option of submitting homework electronically, both sections are required to take exams in person. The NET section was opened late in the registration process because of demand for the course, resulting in a group of students who mostly had not contemplated taking a synchronous Internet course. Nevertheless, grades continued to be indistinguishable between local and distant students.

\section{REINTRODUCING VIDEO TO AUDIOGRAPHIC TEACHING}

By Spring 2000, availability of compressed video technology and higher capacity connections had increased greatly over 1994. Internet video technology, including the RealVideo system from RealNetworks Inc., provides usable video of moderate resolution and refresh rate at data rates 50 to 100 kilobits per second. Many offices have Internet access 
at rates approaching or exceeding one megabit per second, while home access via Integrated Services Digital Network (ISDN), cable modem, and Digital Subscriber Line (DSL) offers service at rates above 100 kilobits per second.

Our administration held the opinion that distance education is more effective with a live video component. A positive influence in this was the ongoing video-based webcasting from Stanford and Berkeley described above. In support of this opinion, we were provided with facilities to expand our synchronous Internet course delivery, adding compressed video to the desktop audiographic system available in our primary distance education classroom. The configuration is shown in Figure 4. The equipment provided included a "follow-me" camera that keeps the video focused on the instructor, a major improvement over our early Mbone teaching configuration. The courses are Senior and Graduate electives in Computer Science. All but one of them is introductory in nature, so the presentations consist largely of lectures intended to introduce new concepts, attended by a wide cross-section of our highly heterogeneous student population. In this environment, video can provide an additional mode of inter-human communication in that the student receives the "body language" of the instructor in addition to the spoken language.

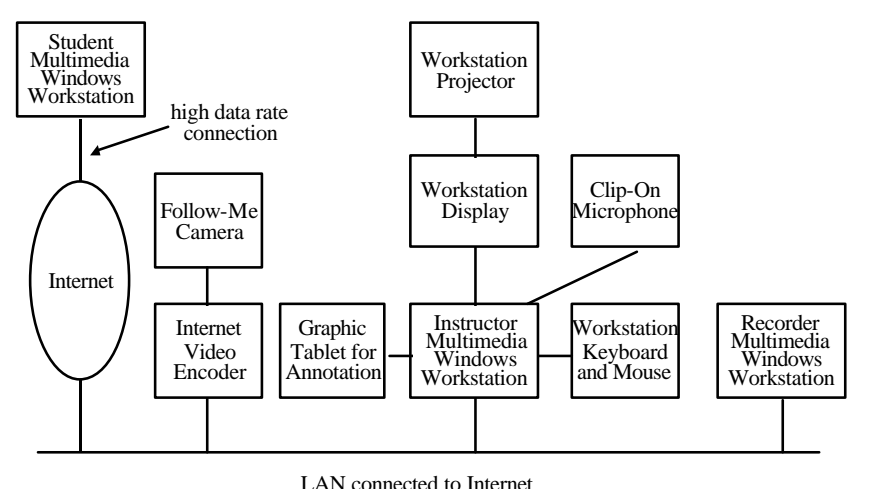

FIGURE. 4

Desktop Audiographic Teaching Configuration Plus Video

We have conducted two semesters of courses with a compressed video component. In the first of these we performed an explicit experiment where a group of nineteen students attended at least one class with video and one class without it. Care was taken by the instructor not to indicate any bias for or against video. As an incentive to participate, students were given a small amount of extra credit (two points out of their grade). However it was made clear that any report, positive or negative, would receive the extra credit. Most students did not have adequate network connections to receive the live video, so we equipped a lab with seven workstations to allow students to participate in classes with video. Coordinating this experiment was not simple, but it gave us valuable data from a controlled environment where each student could compare the experience of classes with and without video.

The responses from this experiment were in accord with our Mbone experience. Even with the instructor consistently visible in the video frame, students reported little value from the video. Some frequency raised points among the nineteen student reports were:

- Nine students stated that video provided no value to them.

- Six students commented that the lack of synchronization between audiographics and video was distracting (whereas buffering delay is an inherent characteristic of compressed Internet video, we chose to keep the audio synchronized with the graphics so students could ask questions in real time).

- Four students commented that the primary use of the video is to let the student know whether class has actually started.

- Two students stated that video helped them to focus on the lecture.

- There was a difference of opinion as to the quality of the video: Two students complained about the quality of the video, while two others stated the video quality was good. Most students did not address this issue.

Overall among the nineteen participating students, the breakout of student opinions regarding use of live video was:

- two students (11\%) were positive about live video

- five students $(26 \%)$ were neutral about live video

- twelve students (63\%) were negative about live video

It appears that some students do benefit from live video. At present our cost to originate live video is low, so we plan to continue providing it. However, our distance education program is moving from experimental to production mode. In Spring 2001 we are teaching five courses using synchronous Internet desktop audiographics, including two NET sections. Three of the courses include a video capability for students who choose to use it. This will allow us to obtain more data on student preference for live video by counting the fraction of students who use this medium. As an initial indication, in Fall 2000 we often had ten to fifteen distant audiographic students, but typically only one or two of these used the video option.

We expect our synchronous distance education offerings will continue to increase. This will drive our administration to an economic decision with regard to offering the live video component. The demand that would be placed on our network facilities by ten remote students using only audiographics would be 200 kilobits per second, whereas audiographics plus video would require around one megabit per second. Thus from a network capacity standpoint we can have five audiographic classes for the cost of one audiographic plus video class. At this time, serving several 
such courses concurrently would place a serious strain on our campus connection to the Internet. Like many universities, GMU has plans to expand its Internet connectivity. That may reduce or eliminate this problem. Ultimately, however, our administration will have to determine whether the additional cost of video is worthwhile in light of the fact that it is considered beneficial by a relatively small percentage of students. A further complicating factor is that, while costs of Internet capacity show a downward trend, the overall cost to the university could actually increase as more students obtain highcapacity connections and elect to receive live video.

\section{CONCLUSIONS}

Our experiment in adding compressed Internet video to synchronous classes delivered by desktop audiographics indicates that, for technical subjects such as computer science and engineering, use of desktop audiographics without video can support a quality synchronous Internet distance education experience. Adding video provides an enhancement to synchronous Internet distance education that is rated as a distinct benefit by only a small fraction of students. Other students are neutral about video or consider it not to be worth the cost of receiving.

The decision to include a video component concerns a quality factor and therefore requires a decision to be made by academic administration. Given a fixed Internet capacity, delivering distance education with only audiographics allows five times as many courses to be supported as would be possible with audiographics plus video, assuming all students choose to receive the video. Nevertheless, as the cost of Internet capacity goes down, it may become more attractive to include a video component in synchronous distance education. Our experience indicates that a primary reliance on desktop audiographics with an optional video feature is a satisfactory compromise at present.

\section{REFERENCES}

[1] Bordeaux, A., D. Sprague, J. M. Pullen and D. Sterling, "Taming the Electronic Frontier: A Distance Education Course for Defense Dependents School Teachers," The Journal of Computing in Teacher Education, Vol. 14 No. 3, Spring 1998

[2] Macedonia, M. and D. Brutzman, "Mbone Provides Audio and Video Across the Internet," IEEE Computer, April 1994

[3] Pullen, J.M. and M. Benson, "ClassWise: Synchronous Internet Desktop Education," IEEE Transactions on Education multimedia CDROM issue, November 1999

[4] Pullen, J. M., "Networking for Distributed Virtual Simulation," Computer Networks and ISDN Systems Journal, Vol. 27, 1994, Elsevier North-Holland

[5] Pullen, J. M., "Synchronous Distance Education Via the Internet," IEEE Frontiers in Education Conference, Salt Lake City, UT, November 1996

[6] Pullen, J. M., "Synchronous Distance Education and the Internet," Internet Society Annual Conference 1998, Geneva, Swi tzerland, July 1998
[7] Pullen, J. M. and E. Norris, "Using A Multi-User Virtual Environment As A Synchronous Teaching Tool," Proceedings of the 1998 Western Simulation Multi-Conference, Society for Computer Simulation San Diego, CA, Vol 30, January 1998

[8] Pullen, J. M., “The Internet Lecture: Converging Teaching and Technology," ACM Special Interest Group on Computer Science Education (SIGCSE) Bulletin Vol 32 No 3 pp 101-104 